\title{
Ulrich Ammon \\ Prinzipien der Sprachwahl im Tourismus, mit Schwerpunkt auf sprachlicher Höflichkeit
}

\begin{abstract}
After defining the concept of ,tourist“ and distinguishing ,inbound tourists“ from ,outbound tourists', the article focuses on questions of politeness towards tourists who are seen and treated as customers of their goals' tourist industry. One possibility of treating them politely is the adequate language choice with respect to their mother tongue, I which they will, as a rule expect to be addressed, if it is a language of considerable international standing (e.g. French or German) or spoken by many tourists in the respective location. Otherwise they will consider it at least more polite to be addressed in a lingua franca, mostly English, than in the local language of the tourist destination. Tourist will find it particularly polite to be addressed in their own mother language if it does not rank among the more international languages. These hypotheses are deduced from well-founded politeness theories based on face-saving and identity assumptions and are illustrated by examples in order to show ways of further empirical corroboration or falsification.
\end{abstract}

Keywords: Concept ,tourist', tourists as customers, language choice for communication, linguistic politeness, face-saving language choice, international languages, mother tongue, lingua franca, foreign tongue

\section{Zum Begriff, Tourist}

Der Hinweis auf die Variabilität des Begriffs ,Tourist ' mag trivial erscheinen. Sich dessen bewusst zu sein, ist allerdings gerade beim $\mathrm{T}$ and hema des vorliegenden Beitrags nicht ganz unwichtig. Daran sollte man auch beim Umgang mit Statistiken denken, die keineswegs immer mit demselben Begriff von ,Tourist' arbeiten (Ammon 2015: 833-836). Ziemlich unproblematisch ist die Unterscheidung zwischen grenzüberschreitenden und $e$ (Reisende innerhalb des Landes). In Bezug auf Erstere wird bei Zählungen meist unterschieden zwischen Einreise- und Ausreise-Touristen (inbound/ outbound). Während manche Angaben zu Touristen Geschäftsleute einbeziehen, beschränken sich andere auf Freizeit- oder Urlaubsreisende. Auf diese hauptsächlich beziehen sich auch die folgenden Überlegungen, also nicht auf Geschäftsreisende,

Ulrich Ammon, Universität Duisburg-Essen, Deutschland, ulrich.ammon@uni-due.de 
Wissenschaftler, Diplomaten, Missionare, Durchreisende, Ausflügler, Langzeitverweilende (,länger als ein Jahr ohne Unterbrechung“; Steinecke 2006: 14), Migranten, Immigranten, Emigranten oder Nomaden.

\section{Höflichkeitsregeln für den Gebrauch von Muttersprache, Fremdsprache und Lingua franca - wenn „der Kunde König“ ist}

Im Folgenden geht es mehr um Forschungsvorschläge, vor allem untersuchenswerte Hypothesen und Illustrationen mit Einzelbeispielen, als repräsentative Daten. Solche finden allerdings verschiedentlich in anderen Beiträgen zum vorliegenden Band. Entsprechend der Beschränkung auf Urlauber im weiten Sinn (Abschnitt 1) ist auch die Frage der Sprachwahl auf diese Gruppe zugeschnitten. Bezüglich Sprachwahl (im Sinne von ,Wahl einer oder mehrerer Sprachen aus verschiedenen Möglichkeiten') kann man unterscheiden zwischen Wahl ,für die Kommunikation“ oder ,für das Sprachlernen'. Im Folgenden geht es vor allem um Erstere, wobei selbstverständlich nur Sprachen gewählt werden können, die schon erlernt wurden: als Mutter-, Zweitoder Fremdsprachen. Unser Blick richtet sich dabei vor allem auf die Abhängigkeit der Sprachwahl von den Sozialbeziehungen zwischen den Kommunikationspartnern. Bezüglich grundlegender Fragen kann man je nach Blickwinkel hinsichtlich menschlichen Handelns von Prinzipien und hinsichtlich Ursachen von Faktoren der Sprachwahl sprechen.

Die vorherrschende oder typische Sozialbeziehung im Tourismus (jedenfalls im hier spezifizierten Sinn) ist die zwischen Gästen (Touristen) und Gastgebern (im Tourismus Beschäftigte, hier mit Schwerpunkt vor Ort). Dabei stellen sich Fragen der Sprachwahl fast nur bei beiderseits verschiedenen Muttersprachen oder staatlichen Amtssprachen, so dass also mindestens eine Seite auf eine Fremdsprache angewiesen ist (oder eine Zweitsprache, die im vorliegenden Zusammenhang nach den Bedingungen ihres Gebrauchs simplifizierend nicht von einer Fremdsprache unterschieden wird). Zwar stellen sich Fragen der Sprachwahl gelegentlich auch bei gleicher Mutteroder Amtssprache der Kommunikationspartner, jedoch ist dies ungewöhnlich oder gilt sogar als kurios: scherzhaft, auf Missverständnis oder Animosität beruhend. So wurde mir z. B. berichtet (freilich ohne sicheren Beleg), dass in der deutschsprachigen Schweiz Deutsche gelegentlich demonstrativ auf Englisch angesprochen würden, allerdings bei anschließendem Umschalten auf die gemeinsame deutsche Sprache. Vielleicht soll mit einer solchen Gesprächseröffnung in einer Fremdsprache aufmerksam gemacht werden auf den nationalen Unterschied oder auf sprachliche Besonderheiten wie das örtlich verbreitete Dialektsprechen oder die eigene nationale Varietät des Deutschen, das Schweizerhochdeutsch (dazu Ammon/Bickel/Lenz 2016). 
Ökonomisch gesehen, ist die Sozialbeziehung zwischen Touristen (im hier spezifizierten Sinn) und Gastgebern ein Sonderfall derjenigen zwischen Kunden (oder Käufern) und Anbietern (oder Verkäufern). Die Touristen kaufen den Urlaubsgenuss von den Verkäufern (Besitzern oder Organisatoren) dieses Vergnügens: in Form von Quartier, Verköstigung, Transport, Sehenswürdigkeiten oder Souvenirs. Dabei sind die Touristen oft in der stärkeren Position - gemäß der Redensart „Der Kunde ist König“. Dies gilt jedenfalls für die typisch marktwirtschaftliche Situation, wo - beim Überschuss an der gehandelten Ware - die Verkäufer um die Gunst der Käufer (Kunden) konkurrieren. Dabei kann diese Konkurrenz zwischen einzelnen Anbietern (z.B. Hotelbesitzern, -angestellten, Inhabern von Souvenirläden usw.) bestehen oder ganzen Ortschaften, Regionen oder Ländern. Sie gewinnt an Schärfe, wenn die Zahl der Touristen begrenzt und die Angebote ungefähr gleichwertig sind. Dann kann die Art des Umgangs mit den Touristen für den Erfolg der Anbieter entscheidend werden, oder können die Anbieter dies zumindest so einschätzen. Eine relevante Komponente dieses Umgangs ist ihr Höflichkeitsgrad, der auch im sprachlichen Handeln zum Ausdruck kommt, und zwar in diversen Komponenten der Sprachpragmatik (z.B. Tonart, Anrede-, Gruß-, Dank-, Entschuldigungsformen usw.) - und unter Umständen eben auch in der Sprachwahl, der Wahl der Sprache zur Kommunikation.

Zum höflichen Handeln, auch spezieller zum sprachlichen, gibt es vielerlei Theorieansätze (siehe z.B. Brown/Levinson ([1978] 1987; Watts 2003; Leech 2014). Dabei zeigen sich auch kulturelle Besonderheiten, aufgrund deren man geradezu von Kulturkreisen der - speziell auch sprachlichen - Höflichkeit sprechen kann. So wurden z.B. markante Unterschiede zwischen westlichen und ostasiatischen Höflichkeitsregeln aufgezeigt - wofür bezüglich spezifisch japanischer Sprachhöflichkeit, als eines vieler möglicher Beispiele, hingewiesen sei auf die Untersuchung von Marui/Nishijima/Noro/Reinelt/Yamashita (1996). Jedoch beschränkt sich der vorliegende Beitrag ganz auf den westlichen Kulturkreis.

Für diesen, sicher auch darüber hinaus (dazu Brown/Levinson [1978] 1987), bietet die Theorie der Gesichtswahrung bzw. der gegenteiligen Gesichtsverletzung einen elementaren Erklärungsansatz, gerade auch für die Sprachwahl. Diese Theorie geht aus von dem Grundgedanken, dass Individuen bei höflichem Verhalten auf Gesichtswahrung ihrer Kommunikationspartner bedacht sind, also darauf, ihr Gesicht nicht zu verletzen. Diese Terminologie ist hergeleitet von der - länderübergreifend gängigen - Redensart, dass jemand in bestimmten Situationen „sein Gesicht verlieren“ kann. Vor allem Erving Goffman ([1959] 2003) hat von diesem Ansatz aus eine viel beachtete Höflichkeitstheorie entwickelt (dazu auch z.B., teilweise weiterführend, O’Driscol 2001a; Brown/Levinson [1978] 1987; Watts 2003: 117-167), die hier aus Platzgründen vereinfacht wiedergegeben wird. Ein Ausgangspunkt ist die Annahme, dass ziemlich alle Menschen zwei Grundbedürfnisse haben, neben sicherlich noch anderen, nämlich die Wahrung ihres positiven und ihres negativen Gesichts. Mit dem 
Erstgenannten ist das Bedürfnis gemeint, von Seiten der Mitmenschen Respekt zu erfahren, mit Letzterem dagegen das Bedürfnis nach persönlicher Freiheit, einem gewissermaßen eigenen Territorium.

In unserem Zusammenhang ist die Wahrung des positiven Gesichts von vorrangiger Bedeutung. Im Hinblick darauf ist jegliche Bezeugung von Anerkennung höflich und jede Unterlassung möglicher Anerkennung weniger höflich oder unhöflich. Solche Bezeugungen oder Unterlassungen können unter bestimmten Umständen auch durch Sprachwahl geschehen. Dabei lässt sich zur Kommunikation selbstverständlich nur eine Sprache wählen, die beide (oder alle) Seiten verstehen; eine andere Wahl, die keine Kommunikation ermöglicht, ist - wenn sie vermeidbar wäre und absichtlich geschieht - grob unhöflich. Bei der Wahl einer für beide Seiten hinreichend verständlichen Sprache ist zu unterscheiden, ob die gewählte Sprache Muttersprache der Gesprächspartner ist oder für sie eine Fremdsprache. Die Symbolik dieser Sprachwahl ist - bis zu einem gewissen Grad - unabhängig von der sachbezogenen Kommunikation. Sie spielt so gut wie keine Rolle, wenn beide Seiten dieselbe Muttersprache haben; ihr Gebrauch gilt dann meist als so selbstverständlich, dass der Gedanke einer Wahl gar nicht aufkommt. Bei verschiedenen Muttersprachen bezeugt dagegen die Seite A Anerkennung gegenüber der Seite B, wenn sie B's Muttersprache gebraucht, obwohl diese für A eine Fremdsprache ist. Sie bekundet damit oder wird so verstanden, dass sie sich B's Muttersprache, als von der eigenen verschieden, bewusst ist und sie als geeignet anerkennt, den Sprachunterschied zu überbrücken. Diese Anerkennung erscheint in der Regel auch als symbolisch für die Wertschätzung der mit der Sprache verbundenen Kultur. Jedenfalls ist diese Wirkung der Sprachwahl A's auf B wahrscheinlicher, als wenn A einfach die eigene Muttersprache gebraucht. Voraussetzung dieser Wirkung ist natürlich, wie schon erwähnt, dass A die Sprache B's hinreichend beherrscht. Andernfalls lässt sich der Verzicht auf den Gebrauch schwerlich als unhöflich bewerten. Allerdings kann die entsprechende Bewertung ausgeweitet werden auf das Erlernen der Sprache, und kann dementsprechend sogar das Nichterlernen (oder Nicht-Erlernt-Haben) als mangelnde Höflichkeit bewertet werden. Als weitere Differenzierungsmöglichkeit sei noch angedeutet, dass darüber hinaus das Erlernen und der Gebrauch einer kleinen Sprache (als Fremdsprache) oft besonders höflich wirkt, höflicher als das Erlernen und der Gebrauch einer großen, weil es genauer auf Gesprächspartner und Sprachgemeinschaft zugeschnitten erscheint. Man vergleiche z.B. die Wirkung des Tschechischsprechens italienischer oder deutscher Touristen in Tschechischen mit der des Französischsprechens in Frankreich. Was wird vor Ort wohl als größere Zuwendung und Höflichkeit empfunden? Mehr dazu in Abschnitt 3.

Des Weiteren lässt sich die symbolische Kommunikation, auch durch die Sprachwahl, unterscheiden von der inhaltlichen, sachbezogenen. Beide können kongruieren, aber auch divergieren. Divergenzen treten oft zutage beim Sprachwechsel (Code Switching): von der Begegnung, die stärker sozialsymbolisch geprägt ist, zur anschließenden, inhaltsbezogenen Kommunikation (z.B. Gruß auf Deutsch oder Italienisch - 
Gesprächsfortsetzung auf Englisch). Naheliegenderweise gelten die entsprechenden Regeln der Sprachwahl auch für andere internationale Begegnungen als im Tourismus, z.B. in der Wirtschaft oder der politischen Diplomatie. Ein Beispiel bot der Fürst von Monaco in Deutschland, bei seinem Besuch der baden-württembergischen Landesregierung: „Fürst Albert spricht den ersten Satz seiner Rede in akzentfreiem Deutsch: ,Es ist eine große Freude für uns, hier in Stuttgart zu sein. Danke.““ Danach fuhr er in seiner Muttersprache, Französisch, fort, das gedolmetscht wurde („Mit ÖkoAntrieb“, Frankfurter Allgemeine Zeitung 11.07.2012: 7). Diese Sprachwahl wurde zweifellos als ausgesprochen höflich gewertet. Entsprechend wirken Kontaktnahme und Gesprächseröffnung seitens gastgebender Tourismusanbieter in der Muttersprache der Touristen.

Allerdings sind die Regeln der Sprachwahl komplizierter als hier darstellbar. Vor allem ist zu warnen vor Übergeneralisierungen. So kann es in Einzelfällen sogar höflich sein, wenn Gastgeber die eigene Muttersprache gebrauchen statt der Muttersprache des Gastes. Dann nämlich, wenn der Gast die Sprache erkennbar gerne gebraucht, womöglich für sein Tourismusziel eigens als Fremdsprache gelernt hat und nun auch anwenden möchte. So wirkt z.B. ein italienischer Hotelier nicht wirklich höflich, wenn er mit einem deutschsprachigen Gast hartnäckig Deutsch spricht, der seine mühsam erlernten Italienischkenntnisse anwenden möchte (dazu: www.goethe.de/ges/spa/prj/sog/fst/de 4622069.htm; abgerufen 15.07.2012). Abgesehen von solchen Sonderfällen ist indes der Gebrauch der Muttersprache gegenüber Kommunikationspartnern, für die sie eine Fremdsprache ist, problematisch. Er verschafft nämlich auf jeden Fall den Muttersprachvorteil im Sinne kommunikativer Überlegenheit. Dieser Vorteil wird akzeptiert, wenn die Sprecher keine andere Wahl haben, weil sie über keine anderen Sprachkenntnisse verfügen. Beherrschen sie jedoch die Muttersprache der Gesprächspartner als Fremdsprache, so wirkt der Verzicht auf deren Gebrauch oft unhöflich.

Eine Notlösung oder Zwischenstufe an Höflichkeit bietet eine Lingua franca, womit in der Regel eine Sprache gemeint ist, die für beide Seiten eine Fremdsprache ist. Man kann dann verdeutlichend auch von einer echten Lingua franca sprechen, im Gegensatz zu einer unechten bei asymmetrischem Gebrauch: Muttersprache des einen und Fremdsprache des anderen Gesprächspartners (echte Lingua franca z.B.: Gebrauch von Englisch zwischen Deutsch- und Französisch-Muttersprachlern; unechte Lingua franca z.B.: Gebrauch von Englisch zwischen Englisch- und Französisch-Muttersprachlern; dazu Ammon 2015: 27-29). Die Wahl einer echten Lingua franca ist insofern höflicher als die Wahl der eigenen Muttersprache, weil sich damit beide Seiten gewissermaßen auf gleicher sprachlicher Augenhöhe (Ohrenhöhe?) begegnen. 


\section{Internationale Stellung von Sprachen als Faktor der Sprachwahl}

Im vorausgehenden Abschnitt ist schon angeklungen, dass auch die Stellung der Sprachen ein Faktor für die Sprachwahl im Tourismus ist, speziell die Stellung in dessen größerem Einzugsgebiet. Allerdings spielt in der heutigen Epoche der Globalisierung sogar die Stellung der Sprachen in der Welt eine Rolle, auch für alle europäischen Touristengebiete. Stark vereinfacht lässt sich die Stellung der Sprachen in der Welt - nach ihrer Verbreitung vor allem als Fremdsprachen und damit ihrer Tauglichkeit zur internationalen Kommunikation - darstellen in einer Rangskala mit 4 Rängen, mit 1) an der Spitze (dazu vor allem de Swaan 2001; auch Ammon 2015: 6374):

- Welt-Lingua-franca (Englisch)

- Internationale Sprachen (alphabetisch: Arabisch, Chinesisch, Deutsch, Französisch, Italienisch, Japanisch, Portugiesisch, Russisch, Spanisch, ...?)

- Nationale Sprachen (Amtssprache ganzer Staaten; ca. 100: z.B. Finnisch, Griechisch, Luxemburgisch usw.)

- Subnationale Sprachen (Regionale Amtssprache oder überhaupt kein amtlicher Status; ca. 2000? - z.B. Baskisch, (West)Friesisch, Romanes usw.).

Die Zahl Letzterer ist fraglich, da bei vielen Zählungen Dialekte als eigenständige Sprachen gezählt werden, anstatt sie als Varietäten von Sprachen diesen jeweils zuzuordnen (dazu Ammon 2015: 31f., 89, 116, 141). Auch ist zu beachten, dass die Übergänge zwischen den 4 hier unterschiedenen Rängen teilweise fließend sind, vor allem von 2) nach 3), was die Auslassungspunkte anzeigen sollen.

Im Tourismus kommen die Sprachen der Rangordnung 1) Englisch, aber auch der Rangordnung 2) in Gebrauch in Gebieten, wo sie weder vorrangige Mutter- noch Amtssprache sind, also als Fremdsprache, und zwar neben den einheimischen Sprachen oder sogar an deren Stelle. In dieser Funktion begegnet man ihnen in den Touristengebieten Europas, allerdings in unterschiedlicher Häufigkeit. Englisch ist so gut wie immer dabei, meist sogar vorherrschend. Sprachen der Gruppe 2) sind geographisch unterschiedlich verteilt, da sich für die Touristengebiete oft nationale oder ethnolinguistische Präferenzen herausgebildet haben. Darüber hinaus finden sich in manchen Touristengebieten auch solche Sprachen als Fremdsprachen, die man eher der Gruppe 3) zuordnen möchte, vor allem Niederländisch, Schwedisch oder Dänisch. Ein Gesamtüberblick über die geographische Verteilung dieser Sprachen als im Tourismus gebrauchte Fremdsprachen wäre ein für die Tourismusplanung vielleicht lohnendes Forschungsprojekt. Eigentlich wäre ihre Pflege eine Aufgabe des touristischen „Destinationsmanagements“, der Gestaltung von Touristenzielen. Jedoch ist diese Aufgabe offenbar ein Stiefkind wissenschaftlicher Tourismusforschung. Sie wird z.B. in dem ansonsten umfassenden Buch von Albrecht Steinecke des Titels 
„Destinationsmanagement“ (2013) gar nicht erwähnt (Ammon 2015: 836), und der Verfasser hat mir brieflich bestätigt, dass sie in der Tourismusforschung bislang kaum beachtet wurde. Jedoch enthalten manche Beiträge zum vorliegenden Band Informationen dazu.

Die Funktion der Fremdsprachen in den Tourismusgebieten ist vielfältig, lässt sich jedoch in übergreifenden Kategorien zusammenfassen - in denen sich sachbezogene Kommunikation und Sozialsymbolik verbinden. Eine dieser Kategorien ist die „sprachliche Landschaft“ (Shohamy/Gorter 2009; bezogen auf Tourismus Kallen 2009; auch Ammon 2015: 855; Nadi, im vorliegenden Band). Die mit diesem Terminus gemeinten öffentlichen Aufschriften dienen einerseits der Orientierung und andererseits der höflichen Begegnung. In Bezug auf Letzteres gibt es seitens der Touristen Erwartungen der Sprachwahl, die abhängig sind von der internationalen Stellung der Sprachen oder auch von der Vorliebe ihrer Sprecher für die jeweilige Destination. So ist es fast schon stereotypisch, dass Briten, aber auch Franzosen vielerorts ihre eigene Sprache berücksichtigt sehen wollen, wogegen z.B. Niederländer oder belgische Flamen dies nur für touristische Lieblingsziele ihrer eigenen Sprachgemeinschaft erwarten.

Entsprechendes gilt für anderen Sprachgebrauch, mündlich oder schriftlich: in Tourismusbüros, Hotelrezeptionen, Restaurants (Speisekarten, Sprachkenntnisse der Bedienungen), bei Sehenswürdigkeiten (z.B. Museen: Beschriftungen, Führungen), Ortsbeschreibungen und Landkarten, in Presseorganen (Zeitungen, Zeitschriften, Bücher) oder auf Webseiten. Offenbar fehlt es aber bisher an entsprechenden umfassenden Beschreibungen von Touristenorten und -regionen. Sema Torgay (1996) hat - vor allerdings geraumer Zeit - die türkische Stadt Antalya hinsichtlich aller Tourismussprachen und ihrer Funktionen vor Ort untersucht, angeregt durch Wolfgang Wölcks (1976) Vorschlag zur Erstellung von Ortsprofilen, wie man seinen Terminus community profiles übersetzen könnte. Entsprechende Untersuchungen ließen sich mutatis mutandis auch auf ganze Regionen oder sogar Staaten ausdehnen. Könnte man in die sprachliche Touristenvorbereitung und -führung (dazu z.B. Costa 2011) auch solche, natürlich jeweils kurze Überblicke einbeziehen, vielleicht an Beispielen bevorzugter Destinationen?

Um die symbolische Funktion der Sprachwahl nicht zu hoch zu bewerten, sei abschließend noch an das universale menschliche Bedürfnis nach gelingender sachbezogener Kommunikation erinnert, das H. Paul Grice ([1975] 1989) im „Kooperationsprinzip“ spezifiziert hat, sowie auch an die damit zusammenhängende, generelle Neigung zu sprachlicher Anpassung (Akkomodation) an die Kommunikationspartner (dazu z. B. Giles 1977). Dabei verbindet sich jedoch das fundamentale Kommunikationsbedürfnis mit Machtverhältnissen (wie zwischen Käufer und Verkäufer) oder auch Anerkennung und Betonung nationaler oder ethnolinguistischer Identität (O'Driscol 2001b; Ammon 2015: 294, 334f.). In dieser Hinsicht können auch lokale und nationale Varietäten einer Sprache bedeutsam werden, die den sprachlichen Landschaften, Speisenkarten usw. ein spezielles Lokalkolorit oder fast schon einen Schuss Exotik 
verleihen (detailliert für Österreich und sein Bundesland Tirol: Dannerer/Franz, im vorliegenden Band). Umgekehrt kann der Verzicht auf regions- oder ortsspezifische Varianten auch heimatstolze Sprachkritiker auf den Plan rufen. So wurde in Österreich der Verzicht auf die eigenen nationalen Varianten verschiedentlich kritisiert. Ein Beispiel ist der Aufruf zum „Kampf an der Sahnefront“ des österreichischen Autors Friedrich Torberg, der sich gegen die „Verdrängung“ österreichischer Varianten der deutschen Sprache (Rahm oder Obers) durch Varianten aus Deutschland (Sahne) richtete, die auch als Folge des von dort einströmenden Tourismus gesehen wurde (http://www.wienerzeitung.at/meinungen/glossen/657106_Wie-Frauen-ihr-Dekolletezum-Hingucker-machen.html - abgerufen 29.01.2018). Auf diese Wirkung des Tourismus aus Deutschland weist auch Rudolf Muhr hin (2017: 263; dankenswerter Hinweis von Rudolf de Cillia).

\section{Literaturverzeichnis}

Ammon, Ulrich (2015): Die Stellung der deutschen Sprache in der Welt. Berlin/München/Boston: Walter de Gruyter.

Ammon, Ulrich/Bickel, Hans/Lenz, Alexandra [1. Aufl. 2004] (2016): Variantenwörterbuch des Deutschen. Die Standardsprache in Österreich, der Schweiz, Deutschland, Liechtenstein, Luxemburg, Ostbelgien und Südtirol sowie Rumänien, Namibia und Mennonitensiedlungen. 2., neu bearb. Aufl. Berlin/Boston: Walter de Gruyter.

Brown, Penolope/Levinson, Stephen C. [1978] (1987): Politeness. Some Universals in Language Usage. Cambridge UK: Cambridge University Press.

Costa, Marcella (2011): Die kommunikative Gattung Touristenführung. Aktivitäten der Wissensvermittlung mit Deutsch als Fremdsprache. In: Reuter, Ewald (ed.): German as a Foreign Language. Sonderheft zum Thema DaF im Tourismus - Tourismus im DaF-Unterricht, 33-54. [Online: www.gfl-journal.de]

de Swaan, Abram (2001): Words of the World. The Global Language System. Cambridge UK: Polity Press.

Giles, Howard (ed.) (1977): Language, Ethnicity and Intergroup Relations. London: Academic Press.

Goffman, Erving [engl. 1959] (2003): Wir alle spielen Theater. Die Selbstdarstellung im Alltag. München: Piper.

Grice, H[erbert] P. [1975] (1989): Logic and conversation. In: Grice, H. P. (ed.) Studies in the Way of Words. Cambridge, MA: Harvard University Press, 22-40.

Kallen, Jeffrey (2009): Tourism and representation in the Irish linguistic landscape. In: Shohamy/ Gorter, 270-283.

Leech, Geoffrey (2014): The Pragmatics of Politeness. Oxford etc.: Oxford University Press.

Marui, Ichiro/Nishijima, Yoshinori/Noro, Kayoko/Reinelt, Rudolf/Yamashita, Hitoshi (1996): Concepts of communicative virtues (CCV) in Japanese and German. In: Ammon, Ulrich/Hellinger, Marlis (eds.) Contrastive Sociolinguistics. Berlin: Walter de Gruyter, 385-409.

Muhr, Rudolf (2017): Zur sprachenpolitischen Situation des Österreichischen Deutsch 2000-2012. In: de Cillia, Rudolf/Vetter, Eva (eds.) Sprachenpolitik in Österreich: Bestandsaufnahme 2011. Informationen Deutsch als Fremdsprache 42 (2-3), 257-305.

O’Driscoll, Jim (2001a): A face model of language choice. In: Multilingua 20 (3), 245-268. 
O’Driscoll, Jim (2001b): Hiding your Difference: How non-global languages are being marginalised in everyday interaction. In: Journal of Multilingual \& Multicultural Development 22 (6), 475-490.

Shohamy, Elana/Gorter, Durk (eds.) (2009): Linguistic Landscape. Expanding the Scenery. New York: Routledge.

Steinecke, Albrecht (2006): Tourismus. Eine geographische Einführung. Braunschweig: Bildungshaus Schulbuchverlage Westermann usw.

Steinecke, Albrecht (2013): Destinationsmanagement. Konstanz/München: UVK Verlagsgesellschaft.

Torgay, Sema (1996): Die Stellung von Deutsch und anderen Fremdsprachen im Tourismus in der Türkei. Unveröff. Magisterarbeit Gerhard-Merator-Universität Duisburg.

Watts, Richard J. (2003): Politeness. Cambridge UK etc.: Cambridge University Press.

Wölck, Wolfgang (1976): Community profiles: An alternative to linguistic informant selection. In: International Journal of the Sociology of Language 9, 43-57. 
DuEPublico

\section{Duisburg-Essen Publications online}

UNIVERSITÄT

DES S SEN R G

offen im Denken

$\mathbf{u b} \mid \begin{gathered}\text { universitäts } \\ \text { bibliothek }\end{gathered}$

Aus: Sociolinguistica (2018), 32(1), S.13 ff.

Dieser Text wird über DuEPublico, dem Dokumenten- und Publikationsserver der

Universität Duisburg-Essen, zur Verfügung gestellt. Die hier veröffentlichte Version der EPublikation kann von einer eventuell ebenfalls veröffentlichten Verlagsversion abweichen.

DOI: $\quad 10.1515 /$ soci-2018-0003

URN: urn:nbn:de:hbz:464-20200721-091602-6

\section{Alle Rechte vorbehalten.}

Dieser Beitrag ist mit Zustimmung des Rechteinhabers aufgrund einer (DFG-geförderten) Allianz- bzw. Nationallizenz frei zugänglich. 